ARTICLE

\title{
Modulating mechanical stability of heterodimerization between engineered orthogonal helical domains
}

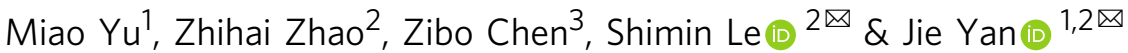

Mechanically stable specific heterodimerization between small protein domains have a wide scope of applications, from using as a molecular anchorage in single-molecule force spectroscopy studies of protein mechanics, to serving as force-bearing protein linker for modulation of mechanotransduction of cells, and potentially acting as a molecular crosslinker for functional materials. Here, we explore the possibility to develop heterodimerization system with a range of mechanical stability from a set of recently engineered helix-heterotetramers whose mechanical properties have yet to be characterized. We demonstrate this possibility using two randomly chosen helix-heterotetramers, showing that their mechanical properties can be modulated by changing the stretching geometry and the number of interacting helices. These helix-heterotetramers and their derivatives are sufficiently stable over physiological temperature range. Using it as mechanically stable anchorage, we demonstrate the applications in single-molecule manipulation studies of the temperature dependent unfolding and refolding of a titin immunoglobulin domain and $\alpha$-actinin spectrin repeats.

\footnotetext{
${ }^{1}$ Mechanobiology Institute, National University of Singapore, Singapore 117411, Singapore. ${ }^{2}$ Department of Physics, National University of Singapore, Singapore 117542, Singapore. ${ }^{3}$ Department of Biochemistry, University of Washington, Seattle, WA 98195, USA. ${ }^{凶}$ email: phyles@nus.edu.sg; phyyj@nus. edu.sg
} 
S pecific heterodimerization systems formed with rapid reversible bonds that can withstand physiological level of mechanical forces (within tens of piconewtons) over physiological time scales (typically within minutes) ${ }^{1-7}$ have a wide scope of applications in biological, biophysical, biomedical, and biomaterial studies ${ }^{8-22}$. For example, substitution of native forcebearing ligand-receptor interactions by mechanically stable heterodimerization systems has revealed how the notch signaling pathway and T-cells can be mechanically activated $17,18,20$. They have also been extensively used as crosslinkers of biopolymers to develop biocompatible hydrogels with unique mechanical prop-

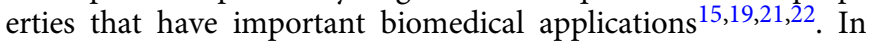
single-molecule mechanical studies of force-dependent structural changes and interactions of biomolecules, these systems have also been utilized as specific mechanically stable molecular anchorage to form single-molecule tethers under force ${ }^{9-14,16,23-28}$.

Currently available mechanically stable and reversible heterodimerization systems can be broadly categorized into several groups: (1) Streptavidin-biotin interaction-based systems ${ }^{8-14,16,24-31}$, (2) Antibody-antigen interaction-based systems ${ }^{12,13,23,25,32,33}$, (3) DNA hybridization-based systems ${ }^{15,17,21}$, and (4) Coiled-coil motif-based systems ${ }^{34,35}$. Streptavidin-biotin systems have been widely used in single-molecule manipulation experiments ${ }^{8-14,16,23-28,31}$ due to the rapid formation of the bond and its high mechanical stability at typical room temperatures ${ }^{36}$. Two representative antibody-antigen systems based on digoxigenin-antidigoxygenin or GST-antiGST interactions have been frequently used as a mechanical anchorage $^{12,13,25,32,33}$. Despite high affinity and high specificity, these antigen-antibody systems have lower mechanical stability than streptavidin-biotin interaction $^{37,38}$. The DNA hybridization-based system has a unique advantage in that its mechanical stability can be programmed over a wide range by modulating the length, sequence and the force geometry applied to the hybrid $17,39,40$. However, as the DNA is a highly negatively charged molecule, potential nonspecific interactions may occur between DNA hybrid and other counter-charged factors. The coiled-coil motif-based system have the advantage of having small molecular weight and can be easily fused to target proteins ${ }^{34,35,41-43}$. The currently coiled-coilbased systems typically rupture at forces of $10-40 \mathrm{pN}$ at high loading rate $\left(\sim 1000 \mathrm{pN} \mathrm{s}^{-1}\right)$ in atomic force microscopy experiment ${ }^{44,45}$, which is comparable to the mechanical stability of digoxygenin-antidigoxygenin complex ${ }^{37}$.

The mechanical stability of heterodimerization systems can vary over a broad temperature range, depending on the nature of the dominant interactions that hold the complex together. In general, the strength of exothermic bonds such as hydrogen bonds decreases as temperature increases ${ }^{46}$, while the strength of endothermic bonds such as many of the entropy-driven hydrophobic interactions increases as temperature increases ${ }^{46}$. The mechanical lifetime of most currently applied heterodimerization systems, such as streptavidin-biotin bond and DNA hybrid, rapidly decreases as temperature increases $29,39,47$, which imposes a strong restriction of their applications in studies involving a significant range of temperature changes.

Therefore, there is a need to develop heterodimerization systems that retain sufficient mechanical stability at physiological temperature range using small protein domains that can be easily fused with the target. Recent studies reported orthogonal heterodimerization between two helix-forming motifs labeled as a and $b$ (Fig. 1a), each being designed to form a helix-hairpin. The interactions between these helix-forming motifs, which result in formation of four-helix bundles (hereafter referred to as helixheterotetramers) with high thermal stability, are optimized for high-affinity orthogonal inter-helix interactions by computational models ${ }^{43,48,49}$ (Fig. 1a). The orthogonality of these heterodimerization systems makes it appealing to develop a variety of mechanically stable heterodimerization systems from them. However, whether these helix-heterotetramers have enough high mechanical stability over physiological temperature range is unclear.

In this study, we quantified the temperature-dependent mechanical stability of two randomly chosen heterodimerization systems (\#13 and \#37) from the reported list ${ }^{49}$ and found that the resulting helix-heterotetramers are sufficiently mechanically stable over physiological temperature range. We further show that mechanical stability of the formed helix-heterotetramers can be changed by modulating the stretching force geometry and the number of interacting helices. We demonstrate the applications of the helix-heterotetramer system as mechanically stable anchorage for single-molecule mechanical manipulation studies of the protein domain unfolding/refolding dynamics and domain stability over physiological temperature range.

\section{Results}

The mechanical stability of the helix-heterotetramer. We developed a single-molecule assay using our magnetic-tweezer setup $^{50-53}$ to directly quantify the loading rate-dependent rupture forces and the force-dependent lifetime of the helixheterotetramers (Supplementary Notes 1-2). Briefly, the singlemolecule construct essentially contains the complementary components of the helix-heterotetramer (hereafter referred to as a and $\mathrm{b}$ ), linked by a long flexible unstructured peptide chain (L) (Fig. 1a left panel, and Supplementary Fig. 1a). The components a and $\mathrm{b}$, each containing two helices a1, a 2 and b1, b2, respectively, are expected to form two helix-hairpins by design ${ }^{49}$. The resulting complex by a and b is, therefore, a helix-heterotetramer. When a and $b$ are separated under force, the linker L keeps them in close vicinity to allow reformation of the complex by dropping the force. In addition, the long linker gives rise to a large extension difference between the separated and associated states of the two components that can be unambiguous distinguished from domain unfolding. Hence, the linker increases both the experimental throughput and the detection accuracy. Importantly, as the peptide linker has a very low bending persistence length, it does not introduce significant mechanical perturbation to the helix-heterotetramers (Supplementary Note 3). The linked a and $\mathrm{b}(\mathrm{a}-\mathrm{L}-\mathrm{b})$ in the construct is spanned between two repeats of the well-characterized titin I27 domain ${ }^{27}$ at each side, which serve as molecular spacers and specificity control. The N- and C- termini of the construct contain a biotin-avi-tag and a spy-tag, respectively, which enables specific tethering for the single-molecule force spectroscopy experiments (Supplementary Notes 1-2).

The linked complementary a and b helix-hairpins can form a helix-heterotetramer at low forces, looping the linker (referred to as the looped state). Rupture of the helix-heterotetramer under force leads to release of the linker, accompanied with a stepwise extension increase (referred to as the unlooped state). As the linker has $>200$ residues, this stepwise extension can be easily differentiated from unfolding of the 89 residues of 127 domain in the handle (Supplementary Note 4$)^{31}$. The looped and unlooped states can be tuned by force (Fig. 1a, right panel). Since the a and b components in the construct are linked in such a way where the force is applied to the N-terminus of the al helix in the a component and the C-terminus of the b2 helix in the $b$ component, we refer the protein construct to as ala2Lb1b2 (left-to-right: N-to-C-termini). The way of force attaching to the construct results in an unzipping force geometry when the helixheterotetramer forms (Fig. la left panel, and Supplementary Fig. 1a); therefore, we refer to the helix-heterotetramer in this construct as $a b^{\text {zip. }}$ 
a
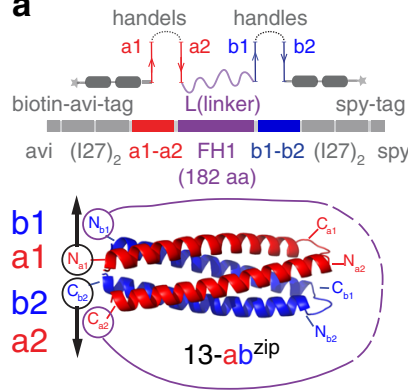

C

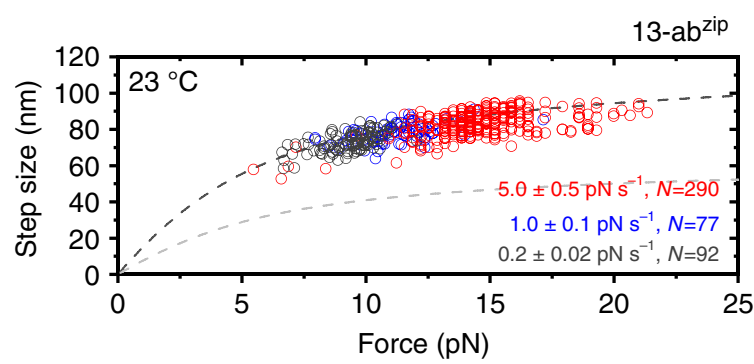

d

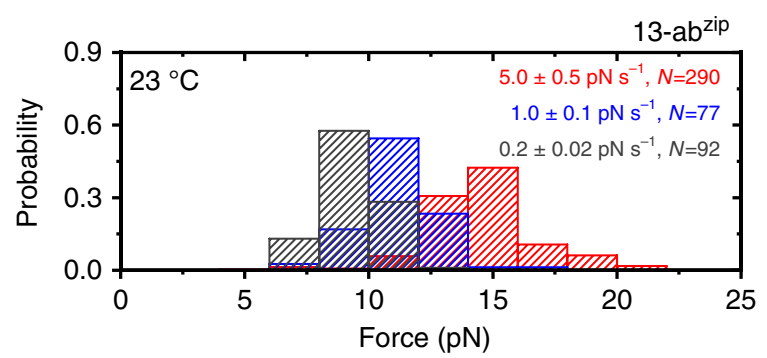

b
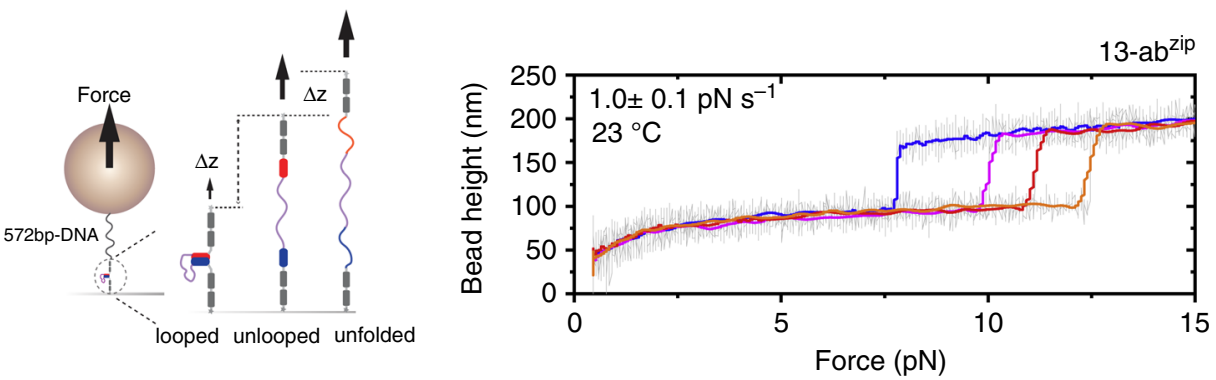

e

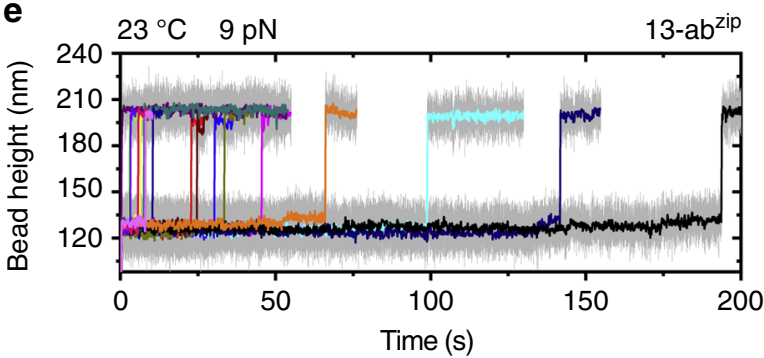

f

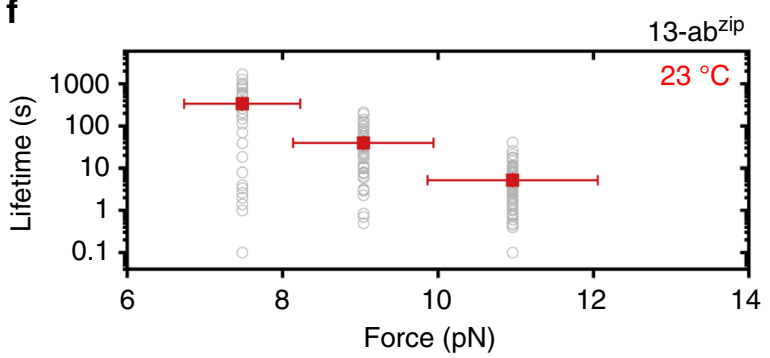

Fig. 1 Single-molecule quantification of the mechanical stability of 13-abzip. a Left-top panel: design of the 13-a1a2Lb1b2-construct. Left-bottom panel: the expected structure of 13-abzip. Black circles and black arrows indicate the force-attaching points and force direction. Purple circles indicate the attachment points of the long linker. Components $a$ and $b$ are helix-hairpins that contains two helices a1\&a2 (red), and b1\&b2 (blue), respectively. Right panel: sketch of a single-molecule construct specifically tethered between a coverslip surface and a superparamagnetic bead with a 572-bp DNA handle (Supplementary Note 2). b Four representative force-height curves of the single-molecule 13-a1a2Lb1b2-construct at $1.0 \pm 0.1 \mathrm{pN} \mathrm{s}^{-1}$. The stepwise bead height jump indicates the force-induced rupturing (unlooping) of the 13-abzip. c The force-step size graph of the force-dependent rupturing (unlooping) transitions of the 13-abzip. The dash curves are the theoretically predicted. The dark gray line is predicted assuming that in the unlooped state a and $b$ components are unfolded into an unstructured peptide chain; the light gray line is predicted assuming that the $a$ and $b$ components remain as folded helix-hairpin structures (Supplementary Note 6 and Supplementary Figs. 4 and 5). d Normalized histograms of the 13-abzip rupture forces. e Representative time traces of the bead height before and after the rupture of the $13-a b^{z i p}$ at $\sim 9 \mathrm{pN}$, where the rupturing events are indicated by the large bead height jump steps. $\mathbf{f}$ Forcedependent lifetimes of the 13-abzip. The solid squares represent the average lifetimes $(\tau)$ obtained by best-fitting of the lifetime histogram to a singleexponential decay function. The hollow gray circles represent individual lifetimes $(N \sim 100$ for each force). The vertical error bars indicate the standard errors of the lifetime obtained by $\frac{\tau}{\sqrt{N-1}}$. The horizontal error bars indicate $10 \%$ of relative force uncertainty resulted from the force-calibration method. For panels b-f: the experiments were performed at $23^{\circ} \mathrm{C}$. For panels $\mathbf{b}$ and $\mathbf{e}$ : 20-points FFT smooth (colored lines) of the raw data (gray lines) are shown. For panels c, $\mathbf{d}$ : the number of data points obtained from $>5$ independent tethers are indicated. Source data are provided as a Source Data file.

In typical single-molecule magnetic-tweezer experiments, a molecule is either subjected to a time-varying force or a constant force, referred to as force-loading or force-clamping experiments, respectively. In the former, the force at which the structural transition of the molecule occurs is recorded, which depends on the force-loading rate. In the latter, the time taken to the transition is recorded, which depends on the level of the applied force. We investigated the force-response of the $13-\mathrm{ab}^{\text {zip }}$ under both force-loading and constant force constraints, where the prefix 13 indicates the \#13 helix-heterotetramer in the reported list ${ }^{49}$.

For the force-loading experiments, we held the construct at sufficiently low force to allow the formation of the looped state and then linearly increased the force with a loading rate, and recorded the rupture force at which unlooping occurred. By repeating the force-loading procedure for sufficient number of cycles, we obtained the distribution of the rupture forces of the 13- $\mathrm{ab}^{\text {zip }}$ helix-heterotetramer at given force-loading rates. We observed characteristic stepwise unfolding signals from the a and b components for the unlooped 13-ab $\mathrm{b}^{\mathrm{zip}}$ construct at forces within $9 \mathrm{pN}$ at a loading rate of $1 \mathrm{pN} \mathrm{s}^{-1}$, associated with step sizes $\sim 12 \mathrm{~nm}$ (Supplementary Fig. 2 and Supplementary Note 5). This result suggests that the helix-forming motifs in $a$ and $b$ indeed form stable helix-hairpins, which further interact with each other to form the helix-heterotetramer. For the quantification of the force-dependent lifetime, after formation of the 13$\mathrm{ab}^{\text {zip }}$ helix-heterotetramer at low forces, we directly jumped to different levels of forces and measured the time duration until unlooping occurred. By repeating the force-jumping procedure for a sufficient number of cycles at multiple force levels, we 
obtained the statistics of the force-dependent lifetime of the 13$\mathrm{ab}^{\text {zip }}$ helix-heterotetramer.

Figure $1 \mathrm{~b}$ shows the representative force-bead height curves of the a1a2Lb1b2-construct from $\sim 1$ to $\sim 20 \mathrm{pN}$ measured with a loading rate of $1 \mathrm{pN} \mathrm{s}^{-1}$. Each colored curve represents one independent force-increase loading cycle. The abrupt stepwise bead height jump in each curve indicates the force-dependent rupturing of the $13-\mathrm{ab}^{\text {zip }}$ helix-heterotetramer and the resulting unlooping. The rupturing of the $13-\mathrm{ab}^{\text {zip }}$ helix-heterotetramer and the unfolding of the separated $a$ and $b$ helix-hairpins typically occurred concurrently because the unfolding forces of the $\mathrm{a}$ and $\mathrm{b}$ helix- hairpins $(<9 \mathrm{pN})$ are smaller than the unlooping forces (Supplementary Fig. 2 and Supplementary Note 5). Here, we note that in such force-loading experiments, due to the small transition distance of I27 $\left(\sim 0.6 \mathrm{~nm}^{27}\right)$, I27 retains its low unfolding rate $\left(\sim 10^{-3} \mathrm{~s}^{-1}\right)$ over the scanned force range up to $50 \mathrm{pN}^{31,54}$. Hence, I27 unfolding was not observed in such forceloading experiments (Supplementary Figs. 3-5 and Supplementary Note 4).

Figure 1c shows force-step size graph of the force-dependent

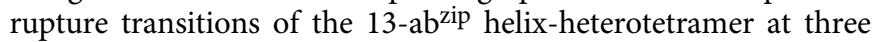
loading rates of $0.2 \mathrm{pN} \mathrm{s}^{-1}$ (dark gray), $1 \mathrm{pN} \mathrm{s}^{-1}$ (blue) and $5 \mathrm{pN}$ $s^{-1}$ (red). The corresponding normalized distributions of the rupture forces shows Gaussian-like distributions with peak values of $\sim 8, \sim 12$, and $\sim 15 \mathrm{pN}$, respectively (Fig. 1d). Figure 1e shows examples of the bead height time traces of the ala2Lb1b2construct at $9 \mathrm{pN}$ during force-clamping experiments (more representative time traces are provided in Supplementary Fig. 6). The $\sim 80 \mathrm{~nm}$ height increase steps are from the force-induced rupturing of 13-ab ${ }^{\text {zip }}$ helix-heterotetramer that causes unlooping and concurrent unfolding of the $\mathrm{a}$ and $\mathrm{b}$ helix-hairpins obtained from different force-clamping cycles. Figure if shows the forcedependent average lifetimes of the $13-a b^{\text {zip }}$ helix-heterotetramer at different forces obtained by fitting the histogram of the measured lifetime data to a single-exponential decay function (Supplementary Fig. 7). The data show that the 13-ab zip helixheterotetramer can withstand forces of 5-12 $\mathrm{pN}$ for seconds to minutes depending on the force level.

Modulating the mechanical stability of the helix-bundles. Using the 13-ab ${ }^{\text {zip }}$ helix-heterotetramer as a starting building block, we explored the possibilities to modulate the mechanical stability of the helix-heterotetramer by manipulating the force geometry. Since molecular complexes under shear-force geometry typically have a stronger mechanical stability than that under the unzipping force geometry ${ }^{54}$, we modified the design of the construct so that the force is applied to the N-terminus of the a2 helix in the a component and the C-terminus of the b2 helix in the b component, leading to a shear-force geometry on the resulting helixheterotetramer (Fig. 2a and Supplementary Fig. 1b). We refer the modified construct to as 13 -a2a1Lb1b2 and the resulting helixheterotetramer to as $13-\mathrm{ab}^{\text {shear }}$.

Figure $2 \mathrm{~b}$ shows the representative force-height curves of 13$\mathrm{ab}^{\text {shear }}$ helix-heterotetramer during force-increase scans with a loading rate of $1 \mathrm{pN} \mathrm{s}^{-1}$. Figure $2 \mathrm{c}$ shows the force-step size graph of the force-dependent rupture transitions of the $13-a b^{\text {shear }}$ helix-heterotetramer at three loading rates of $0.2 \mathrm{pN} \mathrm{s}^{-1}$ (dark gray), $1 \mathrm{pN} \mathrm{s}^{-1}$ (blue) and $5 \mathrm{pN} \mathrm{s}^{-1}$ (red). The corresponding normalized distributions of the rupture forces are shown in Fig. 2d. At all three loading rates, a two-peak distribution was obtained. We reason that the mechanically weaker species could be an intermediate, partially folded structure involving only two or three helices. A possible mechanism that may lead to such intermediate is that the more extended flexible linker in the helixheterotatramer formed by $13-a b^{\text {shear }}$ than that by $13-a b^{\text {zip }}$ could sterically slow down the complete folding process, resulting in such partially folded intermediate. The mechanically stronger species, which is expected to be a helix-heterotetramer involving four helices, is the major form, occupying $70 \pm 5 \%$ of the events.

As the mechanically stronger major species could provide a more stable mechanical anchorage, we quantified the forcedependent lifetime of the 13 -ab $b^{\text {shear }}$ helix-heterotetramer at forces greater than $10 \mathrm{pN}$ (Fig. 2e and Supplementary Fig. 8). The helixheterotetramer can withstand high forces $(15-30 \mathrm{pN})$ over a time scale of seconds to minutes. For comparison, over the similar time scale the $13-\mathrm{ab}^{\text {zip }}$ helix-heterotetramer can only withstand 5-12 pN. Altogether, these results indicate that greater mechanical stability of the helix-heterotetramer can be achieved by changing the geometry of the applied force from unzipping geometry to shearing geometry.

We also explored the possibility of modulating the mechanical stability by changing the number of interacting helices. This was done by creating a construct that contains only three out of the original four helices: the a 2 helix in the a helix-hairpin and the two helices in the b helix-hairpin. This construct is referred to as 13-a2Lb1b2 (Fig. 2f and Supplementary Fig. 1c). In this construct, the $\mathrm{b}$ component forms a stable helix-hairpin structure (Supplementary Fig. 2 and Supplementary Note 5). We expect that the joining of the $a 2$ peptide to the $b$ helix-hairpin could lead to formation of a mechanically stable helix-heterotrimer, referred to as the 13-ahalfb helix-heterotrimer, under a shear-force geometry.

From force-loading and force-clamping experiments, the three helices indeed form a stable complex, but with a weaker mechanical stability than either the $13-a b^{\text {zip }}$ or $13-a b^{\text {shear }}$ helixheterotetramer (Fig. $2 \mathrm{~g}-\mathrm{j}$ and Supplementary Fig. 9). Its rupturing force distribution (Fig. 2i) is similar to the mechanically weaker species observed in Fig. 2d, consistent with the possibility that the mechanically weaker species of $13-\mathrm{ab}^{\text {shear }}$ corresponds to an intermediate structure that involves three interacting helices. This result suggests that it is possible to modulate the mechanical stability via changing the number of interacting helices.

The temperature-dependent mechanical stability. The ability of the helix-heterotetramers to retain their mechanical stability at higher temperature is important for its versatile applications at various temperatures. Hence, we quantified the mechanical stability of the $a b^{\text {zip }}$ and $a b^{\text {shear }}$ helix-heterotetramers at the human body temperature $\sim 37^{\circ} \mathrm{C}$. For the $13-\mathrm{ab}^{\text {zip }}$ and $13-\mathrm{ab}^{\text {shear }}$ helixheterotetramers, the force-loading rate-dependent rupture force distribution (Fig. 3a-b, d-e) and the force-dependent lifetimes (Fig. 3c, f and Supplementary Figs. 10 and 11) show moderate changes from the results obtained at $23^{\circ} \mathrm{C}$. Impressively, the helix-heterotetramer formed by $13-a b^{\text {shear }}$ retained significant mechanical stability at the highest temperature $\left(\sim 47^{\circ} \mathrm{C}\right)$ tested in our experiments (Supplementary Fig. 12). These results suggest that the helix-heterotetramers still have sufficient mechanical stability at $37^{\circ} \mathrm{C}$ which can be used over physiological temperature range.

The formation of helix-heterotetramer is rapid and robust. The ability to rapidly form the helix-heterotetramer is also important for its various applications. Hence, we quantified the rate of the helix-heterotetramer formation. Briefly, we first kept the 13a1a2Lb1b2-construct in the unlooped state at a sufficiently high force, followed by a force jump to a lower value of $1-3 \mathrm{pN}$, and then held the construct at the lower force for a time duration of $\Delta t=2,5,10, \ldots \mathrm{sec}$, to allow the potential formation of the 13$a b^{\text {zip }}$ helix-heterotetramer (Fig. 4a). If the 13-ab $b^{\text {zip helix- }}$ heterotetramer is formed during $\Delta t$, the bead height will be 
a
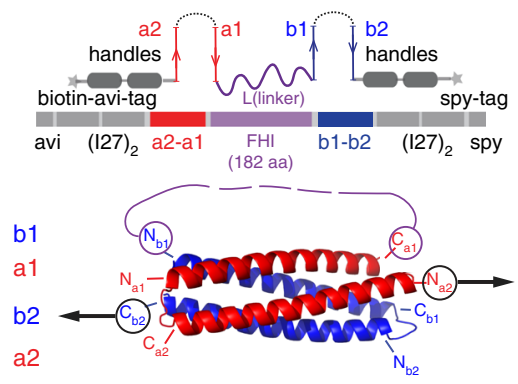

$13-a b^{\text {shear }}$

b

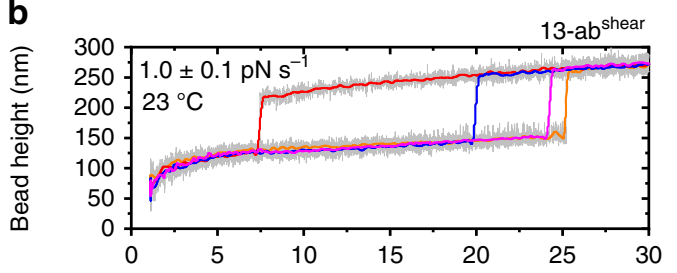

c
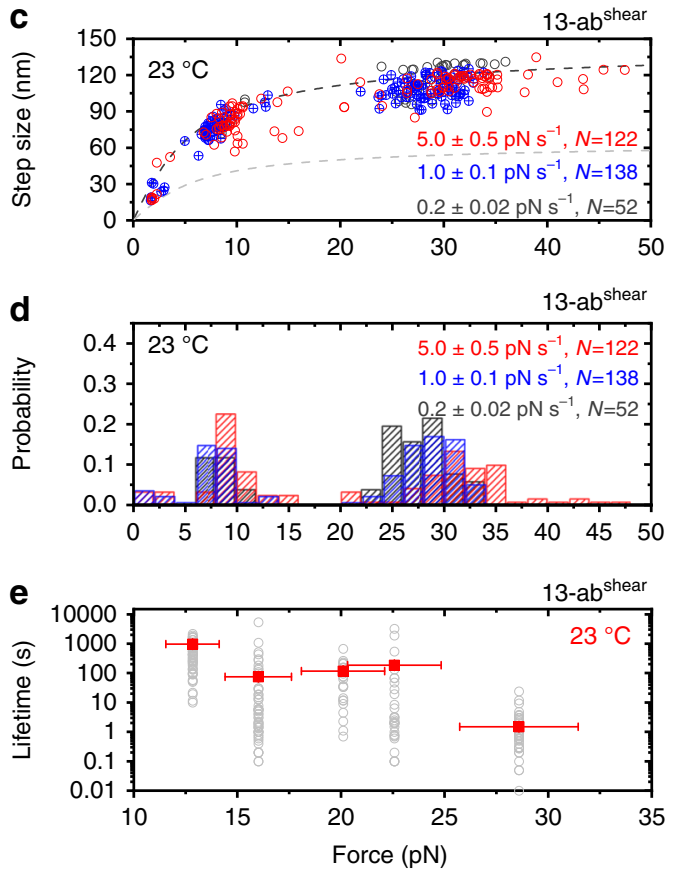

f
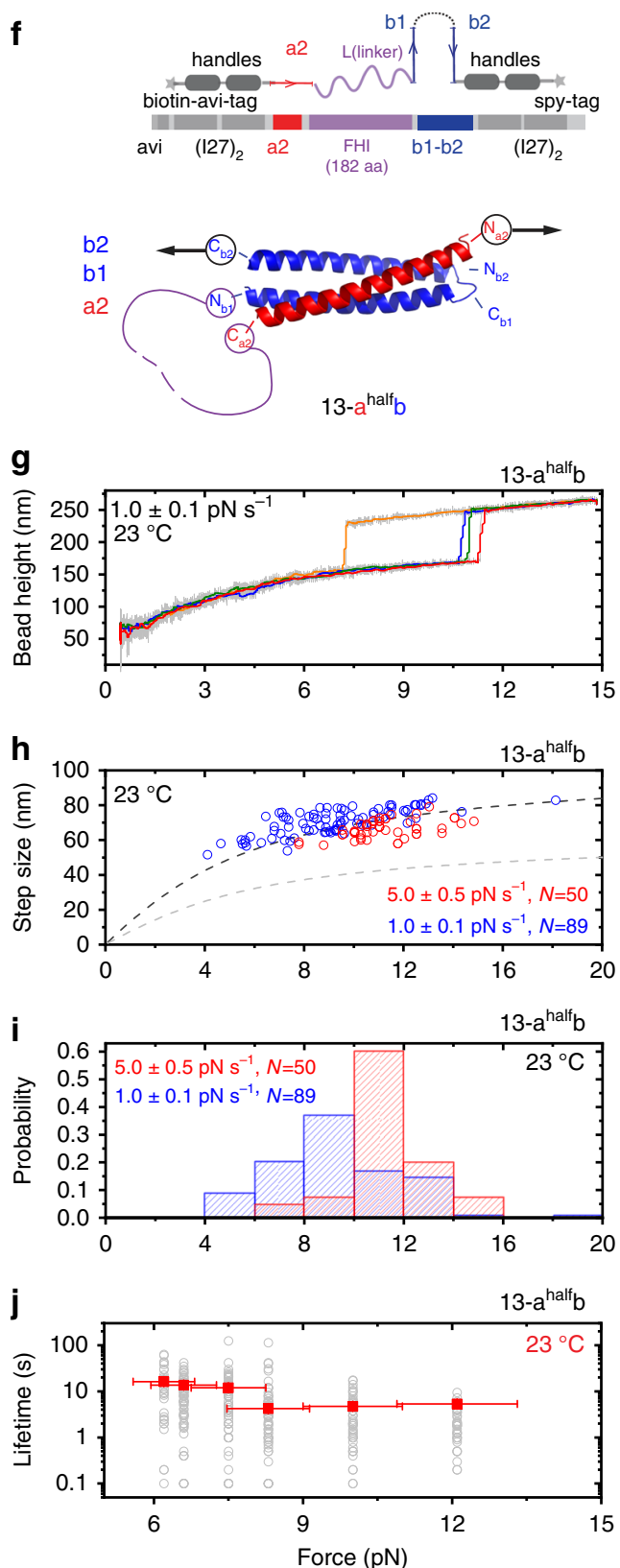

Fig. 2 Modulating the mechanical stability of helix-bundles. a Top panel: design of the 13-a2a1Lb1b2 construct. Bottom panel: The expected structure of the $13-a b^{\text {shear. }}$ b Four representative force-height curves of the 13 -a2a1Lb1b2 construct obtained at $1.0 \pm 0.1 \mathrm{pN} \mathrm{s}^{-1}$. The stepwise bead height jump indicates the force-induced rupturing (unlooping) of the 13-ab ${ }^{\text {shear }}$. c The force-step size graph of the force-dependent rupture transitions of the 13-abshear that causes unlooping of the construct. The dash curves are theoretically predicted. d Normalized histograms of the 13-ab ${ }^{\text {shear }}$ rupture forces. e Forcedependent lifetimes of the 13-ab ${ }^{\text {shear }}$. $\mathbf{f}$ Top panel: design of the 13-a2Lb1b2 construct. Bottom panel: the expected structure of the 13-ahalfb. $\mathbf{g}$ Four representative force-height curves of the single-molecule $13-\mathrm{a} 2 \mathrm{Lb} 1 \mathrm{~b} 2$ construct at $1.0 \pm 0.1 \mathrm{pN} \mathrm{s}^{-1}$. The stepwise bead height jump indicates the forceinduced rupturing (unlooping) of the 13-ahalf b. The force-step size graph of the force-dependent rupturing (unlooping) transitions of the 13-ahalf $b$. The dash curves are theoretically predicted. i Normalized histograms of the 13-ahalf $b$ helix-heterotrimer rupture forces. $\mathbf{j}$ Force-dependent lifetimes of the 13 -a $a^{\text {half }} b$ helix-heterotrimer. For panels $\mathbf{b}$-e and $\mathbf{g}$-j: the experiments were performed at $23^{\circ} \mathrm{C}$. For panels $\mathbf{c}, \mathbf{d}, \mathbf{h}$, and $\mathbf{i}$, the number of data points obtained from $>5$ independent tethers are indicated. Force panels $\mathbf{e}$ and $\mathbf{j}$, the solid squares represent the average lifetimes $(\tau)$ obtained by best-fitting of the lifetime histogram to a single-exponential decay function. The hollow gray circles represent individual lifetimes $(N \sim 100$ for each force) measured in experiments. The vertical error bars indicate the standard errors of the lifetime obtained by $\frac{\tau}{\sqrt{N-1}}$. The horizontal error bars indicate $10 \%$ of relative force uncertainty resulted from the force-calibration method. For panels $\mathbf{b}$ and $\mathbf{g} 20$-points FFT smooth (colored lines) of the raw data (gray lines) are shown. Source data are provided as a Source Data file.

shortened due to the looping of the linker (blue arrows in Fig. 4a). At forces $>1 \mathrm{pN}$, the extension difference between the looped and the unlooped states is $>10 \mathrm{~nm}$. However, due to larger thermal motion of the bead at lower forces, the extension difference might not be visually clear enough at short duration of $\Delta t$
(Supplementary Fig. 13). Therefore, we added an additional force of $\sim 9 \mathrm{pN}$ to distinguish between the looped and the unlooped states of the construct. At this detecting force, the extension difference between two states is $>70 \mathrm{~nm}$ (magenta arrow in Fig. 4a), which allows us to unambiguously determine the state of 
a

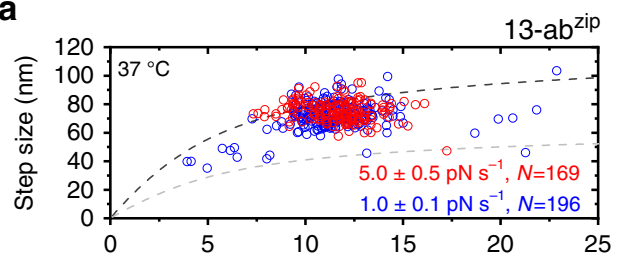

b
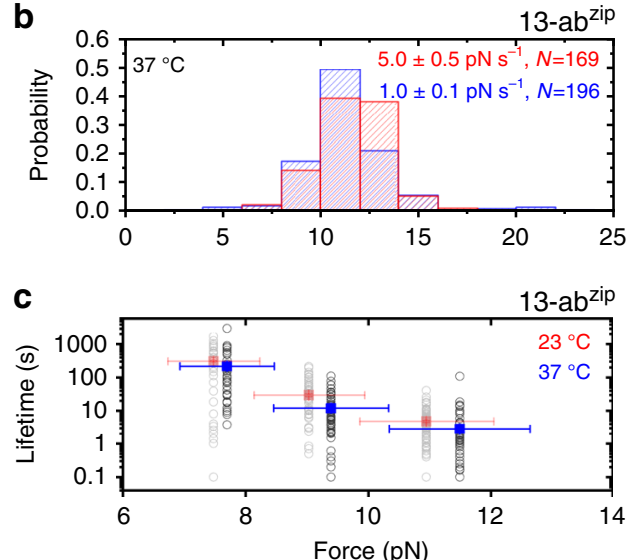

d

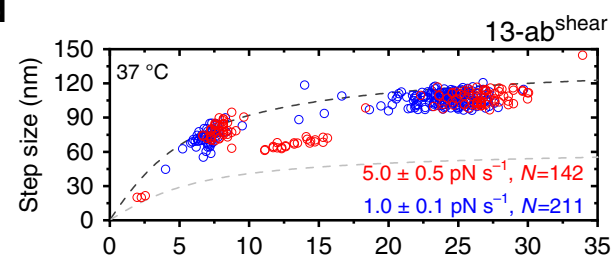

e

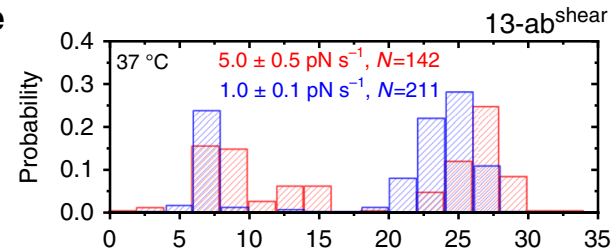

$\mathbf{f}$

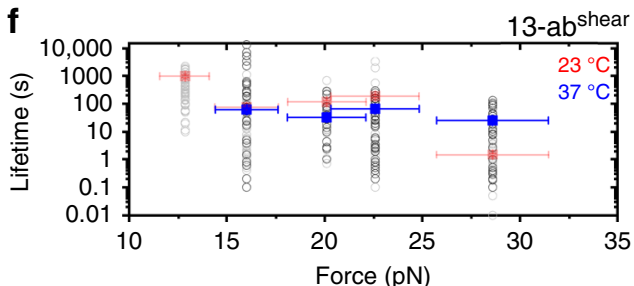

Fig. 3 Temperature-dependent mechanical stability of helix-heterotetramers. a The force-step size graph of the force-dependent rupturing (unlooping) transitions of the $13-a b^{z i p}$ obtained at $37^{\circ} \mathrm{C}$. The dash curves are theoretically predicted. b Normalized histograms of the 13-abzip rupture forces obtained at $37^{\circ} \mathrm{C}$. c Force-dependent lifetimes of the 13 -abzip at $37^{\circ} \mathrm{C}$ (blue) and $23^{\circ} \mathrm{C}$ (red, same as Fig. 1f). d The force-step size graph of the force-dependent rupturing (unlooping) transitions of the 13 -ab shear obtained at $37^{\circ} \mathrm{C}$. The dash curves are theoretically predicted. e Normalized histograms of the 13 -ab ${ }^{\text {shear }}$ rupture forces obtained at $37^{\circ} \mathrm{C}$. f Force-dependent lifetimes of the 13 -ab shear at $37^{\circ} \mathrm{C}$ (blue) and $23^{\circ} \mathrm{C}$ (red, same as Fig. $2 \mathrm{e}$ ). For panels $\mathbf{a}, \mathbf{b}, \mathbf{d}$, and $\mathbf{e}$ : the number of data points obtained from $>5$ independent tethers are indicated. For panels $\mathbf{c}$ and $\mathbf{f}$ : the solid squares represent the average lifetimes $(\tau)$ obtained by best-fitting of the lifetime histogram to a single-exponential decay function. The hollow gray circles represent individual lifetimes $(N \sim 100$ for each force) measured in experiments. The vertical error bars indicate the standard errors of the lifetime obtained by $\frac{\tau}{\sqrt{N-1}}$. The horizontal error bars indicate $10 \%$ of relative force uncertainty resulted from the force-calibration method. Source data are provided as a Source Data file.

a

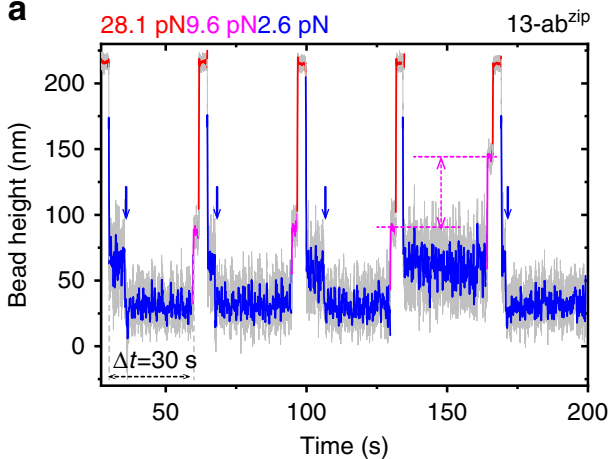

b

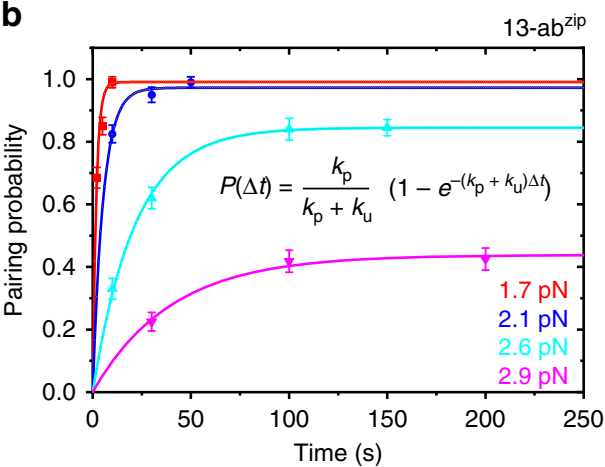

Fig. 4 Force-dependent looping rate of helix-heterotetramer. a An example of the force-clamping procedure to quantify the rate of the formation of the helix-heterotetramer in the linked construct 13-a1a2Lb1b2 at various forces. After jumping from a higher force $(\sim 28.1 \mathrm{pN})$ where the construct is in the unlooped state to a lower force $(\sim 2.6 \mathrm{pN})$, the average looping probability is measured within certain waiting time $(\Delta t=30 \mathrm{~s})$ in multiple cycles. The looping transition is indicated by a stepwise bead height decrease at $\sim 2.6 \mathrm{pN}$ (blue arrows). The magenta dash lines indicate the bead height difference between looped and unlooped states at $9.6 \mathrm{pN}(\sim 70 \mathrm{~nm})$. b Time-dependent looping probability of the construct, $P(\Delta t)$ at different forces indicated by different colors. The vertical error bars indicate standard errors. Colored lines represent the exponential fitting of the pairing probability $P(\Delta t)=\frac{k_{p}}{k_{p}+k_{u}}\left(1-e^{-\left(k_{p}+k_{u}\right) \Delta t}\right)$. Source data are provided in the Source Data file.

the construct prior to the force jump. By repeating the forcejumping assay over one hundred times from more than five tethers, we obtained the probability of the formation of the 13$\mathrm{ab}^{\text {zip }}$ helix-heterotetramer at given lower forces over the waiting time $\Delta t$ (Fig. $4 \mathrm{~b}$ ).

The resulting time-dependent probability of forming the 13$\mathrm{ab}^{\mathrm{zip}}$ helix-heterotetramer at given forces show that at increased forces, the time taken for the looping probability to reach an equilibrium state increased. The looping probability can be fitted to $\mathrm{P}(\Delta t)=\frac{k_{p}}{k_{p}+k_{u}}\left(1-e^{-\left(k_{\mathrm{p}}+k_{\mathrm{u}}\right) \Delta t}\right)$, where $k_{\mathrm{p}}$ and $k_{\mathrm{u}}$ are the rates of looping (13-abzip helix-heterotetramer formation) and unlooping (13-ab ${ }^{\text {zip }}$ helix-heterotetramer rupturing), respectively, which can be determined by fitting to the experimentally measured time evolution of the looping probability (Fig. 4b and Supplementary Table 1). 
The values show that $k_{\mathrm{p}}$ has a strong dependence on force, deceasing over 50 folds when force increased from $1.7 \mathrm{pN}$ $\left(\sim 0.58 \mathrm{~s}^{-1}\right)$ to $2.9 \mathrm{pN}\left(\sim 0.01 \mathrm{~s}^{-1}\right)$. In contrast, $k_{\mathrm{u}}$ is much less sensitive to force over the tested force range, with a value of $\sim 0.005-0.015 \mathrm{~s}^{-1}$ around the force range. The strong force dependence of the looping rate is consistent with a large transition distance as a result from the looping of the flexible linker to reach the transition state. Applying the Arrhenius rate equation, a zero-force looping rate is estimated to be $\sim 15.7 \mathrm{~s}^{-1}$ based on the $k_{\mathrm{p}}(F)$ data points obtained at the four force values (Supplementary Table 1, Supplementary Notes 6-7, and Supplementary Fig. 14). Furthermore, the formation of the $13-a b^{\text {zip }}$ helix-heterotetramer is highly robust, as it can be formed over hundreds of cycles of looping and unlooping. Overall, these results collectively suggest that the $13-\mathrm{ab}^{\text {zip }}$ helixheterotetramer formation is rapid and robust.

To find whether other reported helix-heterotetramer systems have similar properties, we also quantified the $\# 37$ helix-heterotetramer under both unzipping (Supplementary Figs. 15a, 16-21) and shearing force (Supplementary Figs. 15b, 18, 19, 22-24) geometries. The results show that the mechanical stability of the \#37 helixheterotetramer, and its dependence on the force geometry and temperature, are similar to those of the \#13 helix-heterotetramer. Therefore, the principle of modulating the mechanical stability of the complexes could be generally applied to all the helix-heteroteramers in the reported list ${ }^{49}$.

Helix-heterotetramer as a mechanically stable anchorage. We have shown that the \#13 and \#37 helix-heterotetramers can withstand a significant range of mechanical forces over physiological temperature range. The formation of the helixheterotetramers is also rapid and robust. These properties make them appealing candidates to be used as mechanically stable anchorage/crosslinker over physiological temperature range. We demonstrate helix-heterotetramer system's applications as a mechanically stable anchorage in single-molecule studies of force and temperature-dependent protein unfolding/refolding dynamics and protein stability.

In the first example, we used the \#13 helix-heterotetramer to anchor a protein construct (bio-4I27-b) containing four repeats of titin I27 domains (Fig. 5a). In this construct, the I27 domains are spanned between an avi-biotin tag at $\mathrm{N}$-terminus and the b helixhairpin at the C-terminus. The C-terminus of bio-4I27-b construct is specifically tethered to the complementary a helixhairpin immobilized on the bottom coverslip surface under the shear-force geometry (referred as split-13-ab ${ }^{\text {shear }}$, Supplementary Note 2). The biotin-tagged N-terminus is tethered to a superparamagnetic bead via a $572 \mathrm{bp}$ DNA handle (Fig. 5a, left). As previously mentioned, $\mathrm{I} 27$ has a low unfolding rate $\left(\sim 10^{-3} \mathrm{~s}^{-1}\right)$ within $50 \mathrm{pN}^{27,54}$. On the other hand, the 13 -ab ${ }^{\text {shear }}$ helixheterotetramer has a comparable unfolding rate at forces $<10 \mathrm{pN}$ at $23^{\circ} \mathrm{C}$ (Fig. 2e). Therefore, at forces within $10 \mathrm{pN}$, the long lifetime of the $13-\mathrm{ab}^{\text {shear }}$ helix-heterotetramer can potentially be utilized as a mechanically stable anchorage to investigate the mechanical stability of I27.

Figure $5 \mathrm{~b}$ shows six representative time traces of the bead height obtained from six different tethers after a force jump from $<1 \mathrm{pN}$, at which all domains are folded, to a constant force of $\sim 8 \mathrm{pN}$ at $23^{\circ} \mathrm{C}$. In each time trace, four stepwise increases of the bead height are observed, corresponding to unfolding of the four I27 repeats. The step sizes are distributed around $14.9 \pm 1.0 \mathrm{~nm}$ (mean \pm standard deviation), consistent with the release of $\sim 89$ residues of I27 into a disordered polypeptide (Supplementary Note 6 and Supplementary Figs. 4 and 5). Refolding of the unfolded domains was not observed at this force, because the force is greater than the equilibrium critical force of $\mathrm{I} 27(\sim 5.4 \mathrm{pN})$ at $23^{\circ} \mathrm{C}$ as reported previously ${ }^{27,31}$. At this force, the I27 domain has very slow unfolding rate, resulting in long experimental time duration of more than one and half hour for each experiment. This example demonstrates that the long lifetime of the split-13-ab ${ }^{\text {shear }}$ helix-heterotetramer under shear-force geometry over physiological force range, typically a few $\mathrm{pN}$, provides a specific anchoring method to support long duration of single-molecule experiments.

After unfolding, I27 domain exists in a highly disordered state that carries a larger conformational entropy ${ }^{27}$. Therefore, increased temperature is expected to decrease the domain stability. In order to probe the thermodynamic properties of I27, we increased the temperature to $37^{\circ} \mathrm{C}$ and measured the equilibrium unfolding and folding transitions, which were used to calculate the folding energy of $\mathrm{I} 27$. Figure $5 \mathrm{c}$ is a representative time trace of the bead height obtained at $\sim 3.5 \mathrm{pN}$ showing reversible unfolding and folding transitions. From the trace, we obtained the probability distributions of bead height during dynamic unfolding and refolding of the four I27 domains. The probability of having $n$ unfolded I27 domains in four independent I27 repeats, $P_{4}(n)$, can be directly read out from the bead height distribution peaks (Fig. $5 c$, right panel). It follows the binomial distribution: $P_{4}(n)=C_{4}^{n} p^{n}(1-p)^{4-n}$, where $C_{4}^{n}=\frac{4 !}{n !(4-n) !}$ is the binomial coefficient. The single-free parameter $p$ denotes the probability of an I27 domain in the unfolded state at this force.

Fitting of the binomial distribution to the normalized probability of the number of unfolded $\mathrm{I} 27$ repeats, we determined that $p(F) \sim 0.27$ at $3.5 \mathrm{pN}$ at $37{ }^{\circ} \mathrm{C}$ (Fig. $5 \mathrm{c}$ ). The value of $p(F)$ is related to the force-dependent free energy difference between the unfolded and folded states, $\Delta G(F)$, by $\Delta G(F)=\Delta G_{0}+\Delta \phi(F)=-k_{\mathrm{B}} T \ln \frac{1-p(F)}{p(F)}$. Here, $\Delta G_{0}$ is the zeroforce folding energy of the I27 domain, $\Delta \phi(F)=\int_{0}^{F}\left(x_{\mathrm{u}}(f)-\right.$ $\left.x_{\mathrm{f}}(f)\right) \mathrm{d} f$ is the force-dependent conformational free energy difference between the unfolded state and the folded state ${ }^{27,31}$. Using the equation and based on the measured $p(F)$ and forceextension curves of 127 in the folded and unfolded states (Supplementary Note 6), we obtained $\Delta G_{0}=-4.9 \pm 0.4 k_{\mathrm{B}} T$. The critical force $F_{c} \sim 3.9 \mathrm{pN}$ at which the unfolded and folded states have equal probabilities, is also obtained by $\Delta G_{0}+\Delta \phi\left(F_{c}\right)=0$. Comparing these values with those obtained at $23{ }^{\circ} \mathrm{C}^{27,31}$ (Table 1 ), it clearly shows that when temperature increases from 23 to $37^{\circ} \mathrm{C}, \Delta G_{0}$ of I27 increases significantly by more than $3 k_{\mathrm{B}} \mathrm{T}$ (i.e., thermodynamic stability decreases) and correspondingly the equilibrium critical force decreases by $\sim 1.5 \mathrm{pN}$.

In the above example, the I27 domain has a typical $\beta$-sheet structure. In order to probe the thermodynamic properties of protein domains with different ternary structures, such as $\alpha$-helix bundles, we created another protein construct (bio-6SR-b) that contains six repeats of the fourth $a$-actinin spectrin-repeat (SR) domain ${ }^{55}$, spanned between an avi-biotin tag at $\mathrm{N}$-terminus and the $b$ helix-hairpin of the \#13 helix-heterotetramer system at the C-terminus. Each SR domain is formed with three $\alpha$-helices bundled together. Figure 6 a shows representative time traces of the bead height obtained from six independent tethers, each containing six stepwise domain unfolding steps. The step sizes are distributed around $\sim 17.9 \pm 1.0 \mathrm{~nm}$, consistent with the release of $\sim 104$ residues of SR4 into a disordered polypeptide (Supplementary Note 6 and Supplementary Figs. 4 and 5). Using similar approach to quantify the temperature-dependent thermal stability of I27, we quantified the temperature-dependent thermal stability of the spectrin-repeat based on equilibrium two-state transitions over a temperature range from $23^{\circ} \mathrm{C}$ to $31^{\circ} \mathrm{C}$ (Fig. $6 \mathrm{~b}-\mathrm{d}$ and 
a

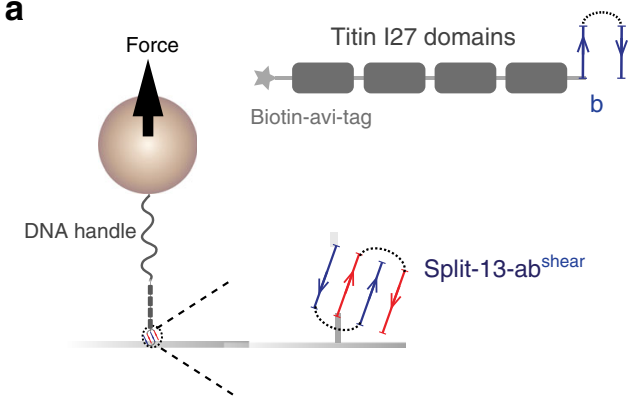

b

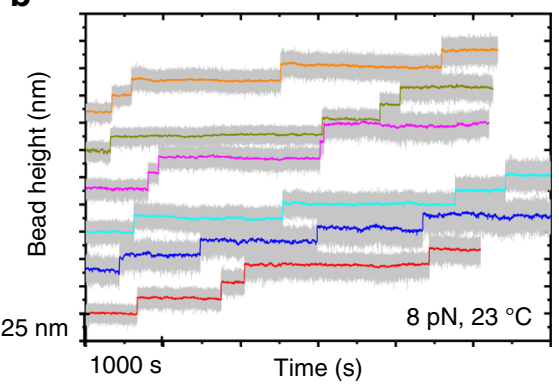

C

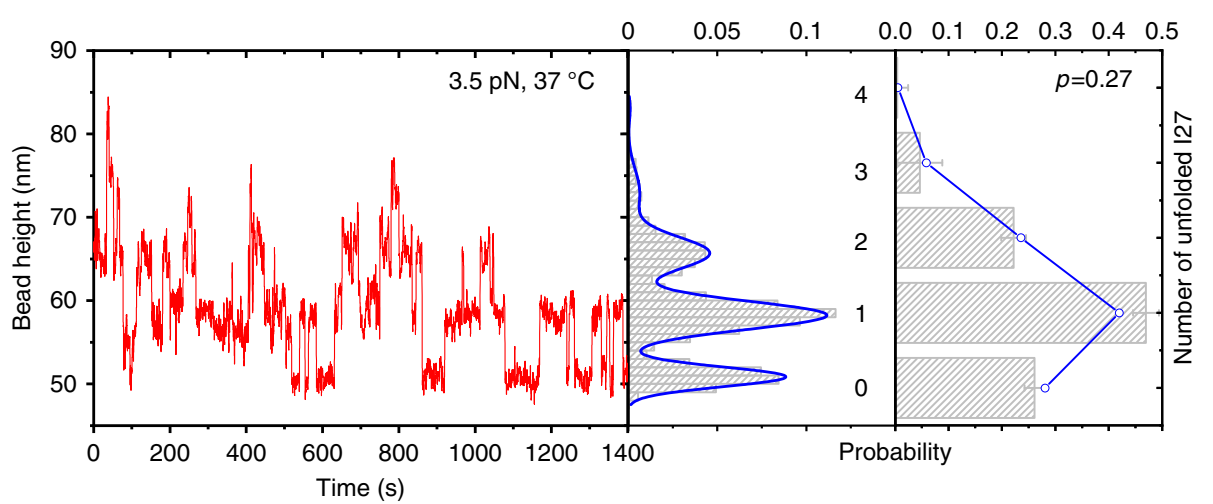

Fig. 5 Temperature-dependent titin $\mathbf{1 2 7}$ unfolding/refolding dynamics. a Experimental design of utilizing the split-13-ab ${ }^{\text {shear }}$ helix-heterotetramer to anchor a protein construct (bio-4I27-b) that contains four repeats of titin 127 domains to coverslip surface. The I27 domains are spanned between an avibiotin tag at N-terminus and the \#13-b helix-hairpin at the C-terminus. The bio-4I27-b construct is specifically tethered to the \#13-a helix-hairpin coated bottom surface via formation of split-13-ab ${ }^{\text {shear }}$ complex, and the N-terminus is tethered to the DNA-coated magnetic bead through biotin-streptavidin interaction (details are provided in Supplementary Note 2). $\mathbf{b}$ Six representative time traces of the bead height obtained from six independent tethers under a constant force of $\sim 8 \mathrm{pN}$ at $23^{\circ} \mathrm{C}$. The four stepwise extension increase events with a step size of $\sim 15 \mathrm{~nm}$ indicate 127 unfolding. Colored lines are 200 points FFT smooth of the raw data (gray). c Left panel: a representative time trace of the bead height (20-points FFT smoothed) under a constant force of $\sim 4 \mathrm{pN}$ at $37^{\circ} \mathrm{C}$. The stepwise extension increase/decrease with step sizes of $\sim 10 \mathrm{~nm}$ indicates unfolding/refolding of the 127 domains. Middle panel: the corresponding probability distributions of the bead height. Blue line is the multiple peak Gaussian fitting of the bead height probability distributions. Right panel: The bar graph shows the probability of having $n$ unfolded 127 domains in the four 127 domains obtained from the bead height distribution in the middle panel. The error bar indicates the standard error, which is obtained through multiple peak Gaussian fitting of the bead height probability distributions. Blue curve indicates the fitting of the bar graph to the binomial distribution, from which the probability of the unfolded state of a single 127 domain at this force is determined to be: $p(F) \sim 0.27$ at $37^{\circ} \mathrm{C}$. Source data are provided in Source Data file.

Table 1 Thermal stability of the $\alpha$-actinin 1 SR4 and titin I27 domains.

$\alpha$-Actinin 1 SR4

$F(\mathrm{pN})$

$T\left({ }^{\circ} \mathrm{C}\right)$

$\mathrm{p}(F)$

$\Delta \phi(F)\left(K_{\mathrm{B}} T\right)$

$\Delta G(F)\left(K_{\mathrm{B}} T\right)$

$\Delta G_{0}\left(K_{\mathrm{B}} T\right)$

$F_{c}(\mathrm{pN})$
$5.7 \pm 0.57$

23

$0.091 \pm 0.002$

$10.90 \pm 2.03$

$-2.30 \pm 0.02$

$-13.2 \pm 2.0$

$6.3 \pm 0.5$
$5.7 \pm 0.57$

27

$0.283 \pm 0.004$

$10.38 \pm 2.01$

$-0.93 \pm 0.02$

$-11.3 \pm 2.0$

$6.0 \pm 0.6$

$5.7 \pm 0.57$
29
$0.531 \pm 0.005$
$10.31 \pm 1.99$
$0.12 \pm \pm 0.02$
$-10.2 \pm 2.0$
$5.7 \pm 0.6$

Titin 127

$\begin{array}{lll}5.7 \pm 0.57 & 4.5 \pm 0.45 & 3.5 \pm 0.35 \\ 31 & 23 & 37 \\ 0.722 \pm 0.008 & 0.131 \pm 0.004 & 0.272 \pm 0.004 \\ 10.15 \pm 1.96 & 6.44 \pm 1.1 & 3.95 \pm 0.7 \\ 0.95 \pm 0.04 & -1.89 \pm 0.05 & -0.98 \pm 0.04 \\ -9.2 \pm 2.0 & -8.3 \pm 1.0 & -4.9 \pm 0.7 \\ 5.4 \pm 0.6 & 5.4 \pm 0.4 & 3.9 \pm 0.3\end{array}$

For $\alpha$-actinin 1 SR4 domain, experiments were performed at four different temperatures $\left(23,26,29\right.$, and $\left.31^{\circ} \mathrm{C}\right)$ at a constant force of $5.7 \mathrm{pN}$. For titin 127 domain, experiments were performed at $37{ }^{\circ} \mathrm{C}$ at a constant force of $3.5 \mathrm{pN}$. The data for titin 127 at $23^{\circ} \mathrm{C}$ at a constant force of $4.5 \mathrm{pN}$ were taken from previous publications 27,31 for comparison. The error bars (s.t.d.) for $p(F)$ are obtained by bootstrap analysis; for $\Delta \Phi(F)$ are obtained by error propagation from the $10 \%$ of relative uncertainty in force-calibration ("Methods"); for $\Delta G(F)$ are obtained by error propagation from the error bars of $p(F)$; for $\Delta G_{0}$ are obtained by error propagation from the error bars of $\Delta \Phi(F)$ and $\Delta G(F)$; for $F_{c}$ are obtained by error propagation from the error bars of $\Delta G_{0}$. Source data are provided as a Source Data file.

Table 1), and revealed that the stability of the spectrin-repeat is negatively influenced by increased temperatures.

\section{Discussion}

In summary, by mechanically characterizing two engineered helix-heterotetramers reported in ref. ${ }^{49}$ using single-molecule construct assays, we show that their mechanical stabilities are retained over physiological temperature range. Their mechanical stability can be modulated by changing the force geometry and by changing the number of interacting helices. As the two helixheterotetramers investigated in this study were randomly chosen from the list of the six biochemically characterized orthogonal helix-heterotetramers ${ }^{49}$, the retained mechanical over physiological temperature range could be a shared property of several helix-heterotetramers of the reported list ${ }^{49}$. The principle can be extended to other reported helix-heterotetramers, highlighting the potential for development of a variety of helixheterotetramers with different mechanical stability for specific linking of protein domains. 
a

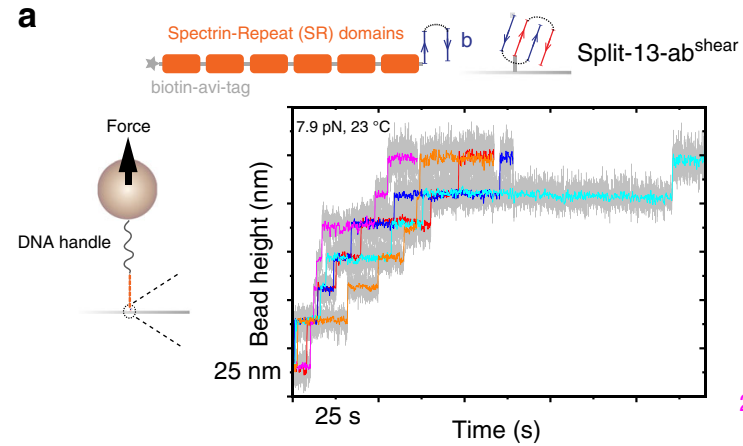

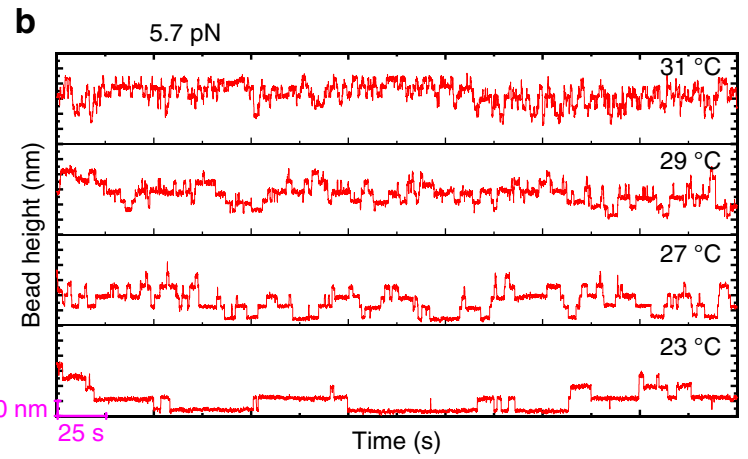

d

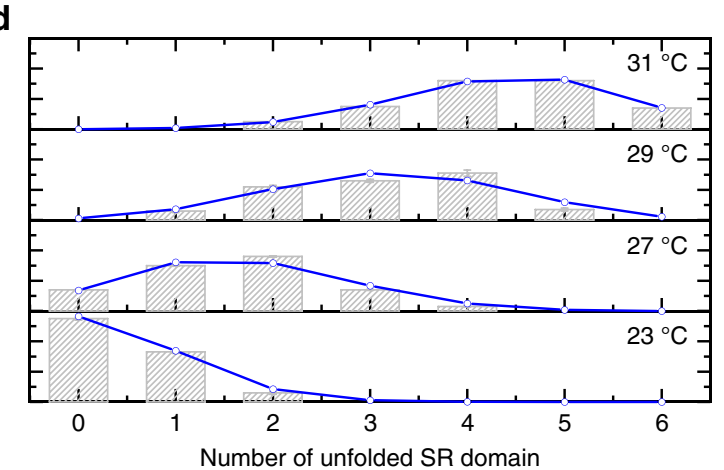

Fig. 6 Temperature-dependent $\boldsymbol{\alpha}$-actinin SR4 unfolding/refolding dynamics. a Top panel: experimental design of utilizing the split-13-ab ${ }^{\text {shear }}$ to anchor a protein construct (bio-6SR-b) that contains six repeats of the fourth spectrin-repeat domain to glass surface. The six SR domains are spanned between an avi-biotin tag at N-terminus and the \#13-b helix-hairpin at the C-terminus. The bio-6SR-b construct is specifically tethered to the \#13-a helix-hairpin coated bottom surface via formation of split-13-ab ${ }^{\text {shear }}$ complex, and the N-terminus is tethered to the DNA-coated magnetic bead through biotin-streptavidin interaction (Supplementary Note 2). Bottom panel: five representative time traces of the bead height obtained from five independent tethers under a constant force of $\sim 7.9 \mathrm{pN}$ at $23^{\circ} \mathrm{C}$. The six stepwise extension increase events with a step size of $\sim 25 \mathrm{~nm}$ in each time trace indicate SR unfolding. Colored lines are 20-points FFT smooth of the raw data (gray). b Representative time traces of the bead height (20-points FFT smoothed) under a constant force of $\sim 5.7 \mathrm{pN}$ at multiple temperatures $\left(23,27,29\right.$, and $31^{\circ} \mathrm{C}$ ). The stepwise extension increases and decreases with step sizes of $\sim 18 \mathrm{~nm}$ indicate unfolding/ refolding of the SR domains. $\mathbf{c}$ The resulting probability distributions of the bead height. The numbers of unfolded spectrin-repeat domains at corresponding bead height are indicated. Blue line is the multiple peak Gaussian fitting of the bead height probability distributions. $\mathbf{d}$ The bar graph shows the probability of having $n$ unfolded SR domains in the six SR domains obtained from the bead height distribution in $\mathbf{c}$. The error bar indicates the standard error, which are obtained through multiple peak Gaussian fitting of the bead height probability distributions. Blue curve indicates the fitting of the bar graph to the binomial distribution. Source data are provided in Source Data file.

Our results demonstrate that, through modulating the force geometry, the force-dependent lifetime of the two tested helixheterotetramers can be tuned to cover the typical physiological force range over typical physiological time scale ${ }^{1-7}$. The mechanical stability of the helix-heterotetramers is greater under shearing force geometry than under unzipping force geometry, consistent with recent theoretical studies ${ }^{40,54}$. Impressively, despite their small size ( 150 a.a. in the complex), a single helixheterotetramer under shear-force geometry can survive for minutes at forces below $20 \mathrm{pN}$, which reaches the mechanical strength of antibody/antigen complexes that are much larger in size. We also show that mechanical stability is positively dependent on the number of interacting helices, suggesting another potential route to tune the mechanical stability via increasing the range of possible interacting helices. Sequence modifications of the helixheterotetramers, which were not explored in this study, provide another possibility. However, for the helix-heterotetramers developed in ref. ${ }^{49}$, sequence modifications may be unwantedly destabilizing considering that these helix-heterotetramers have been optimized for high stability ${ }^{49}$. It is important to note that our conclusions are based on the two tested helixheterotetramers. Therefore, a more comprehensive understanding of how the mechanical stability of helix-heterotetramers depend on force geometry, the number of interacting helices and the sequence modifications warrants future studies.

One exciting feature is that the two helix-heterotetramers investigated in this study retain significant mechanical stability at 23 and $37^{\circ} \mathrm{C}$, which spans the typical physiological temperature range. The temperature dependence of the mechanical stability of the two helix-heterotetramers is related to the molecular details of the interactions on the transition pathways during mechanical rupture of the complexes. The very high thermodynamic stability of the two tested helix-heterotetramers characterized by melting temperatures $>95^{\circ} \mathrm{C}^{49}$ suggest that they contain a large fraction of endothermic hydrophobic interfaces distributed throughout the helix-heterotetramers. Hence, the moderate influence of the temperature on the mechanical stability of the two tested helixheterotetramers when temperature increases from 23 to $37^{\circ} \mathrm{C}$ can be explained by the presence of a significant fraction of the endothermic hydrophobic interactions on the transition pathways. Based on this principle, we propose that the key to decrease the temperature sensitivity of the mechanical stability of a protein complex is to design a transition pathway containing significant amount of endothermic hydrophobic interactions.

The helix-heterotetramers that retain significant mechanical stability over physiological temperature range is a highly 
advantageous property for their use as force-bearing adapter molecules in a broad range of applications. In this study, we demonstrated the application of the heterotetramer under shearing force geometry as a mechanically stable anchorage to investigate the temperature-dependent stability of two different types of protein domains (titin I27 and $\alpha$-actinin spectrin-repeat) using magnetic-tweezers. We probed the equilibrium unfolding and folding transitions of these protein domains at different temperatures from 23 to $37^{\circ} \mathrm{C}$, from which we determined their thermal stability (i.e., $\Delta G_{0}$ ) at each temperature. Our results show that for these protein domains, their thermal stability decreases as temperature increases, suggesting that the folding of these domains is overall an exothermic reaction involving predominantly enthalpy driven processes of folding. This approach can be broadly applied to investigate other protein domains to obtain insights into the physical nature of the folding processes.

Besides serving as a mechanically stable molecular anchorage for single-molecule manipulation studies, the helix-heterotetramers have many other potential applications. The specific interaction between $\mathrm{a}$ and $\mathrm{b}$ components enable their use as mechanically stable molecular crosslinkers, as they can be fused to respective target molecules (Supplementary Fig. 25). The orthogonality and the potential programmability of the multiple sets of the complementary $\mathrm{a}$ and $\mathrm{b}$ helix-pairs promise a wide scope of potential applications in developing functional materials. For example, the helix-heterotetramers are promising reversible crosslinkers of biopolymers such as polyprotein peptides or nucleic acids for engineering self-healing hydrogels $15,21,22$ or other actin/microtubulebased functional materials ${ }^{56-59}$ with variable mechanical properties over physiological temperature range.

The helix-heterotetramers have broad applications in mechanotransduction studies, serving as a specific building block for various specific mechanical linkages. The forces transmitted on force-transmission supramolecular linkages in the cells are in the order of a few $\mathrm{pN}^{1,3,4,6}$. At the cell-extracellular matrix and cell-cell adhesions, the forces transmitted on single molecules are also in similar range ${ }^{2,5,7}$. In addition, numerous known mechanosensing domains in different force-bearing proteins, such as talin, $\alpha$-actenin and dystrophin, have been shown being unfolded by forces from a few $\mathrm{pN}$ to $30 \mathrm{pN}$ at physiologically relevant loading rates ${ }^{28,55,60}$. Owing to the typical low loading rates exerted to force-transmission proteins by cells ${ }^{61}$, forces generated from live cells could unfold many protein domains, including the ones with high mechanical stability ${ }^{62-64}$. Therefore, the mechanical stability of these helix-heterotetramers is able to support studies of a wide force range of important mechanosensing proteins and mechanotransduction processes in live cells. For example, by genetically linking the complementary a and $b$ components with actin binding domains (ABD), one can build artificial actin crosslinkers to organize actin filaments in many different ways. The crosslinking strength of such artificial actin crosslinkers can be tuned by modulating the stability of the helix-heterotetramers and by using different ABDs derived from different actin binding proteins. The length and the flexibility of the artificial actin crosslinkers can also be modulated by inserting protein spacers between the $\mathrm{a}$ or $\mathrm{b}$ component and ABDs. The highly variable design of such artificial actin crosslinkers may replace or compete with endogenous actin crosslinkers such as filamin $\mathrm{A}$ and $\boldsymbol{\alpha}$-actinin to modulate cell behaviors ${ }^{65-67}$.

\section{Methods}

Plasmids preparation and protein expression. The DNA fragments encoding al, a2, b1, b2 helices, the long flexible linker (FH1 domain (residues 582-764 of DIAP1_HUMAN)), the four repeats of titin I27 domains (4I27), and the six repeats of the fourth $\alpha$-actinin spectrin-repeat domains (6SR), as well as the avi-tag
(GLNDIFEAQKIEWHE) and spy-tag(AHIVMVDAYKPTK) ${ }^{68}$ were synthesized by GeneArt/gBlock. The corresponding DNA fragments were then sub-cloned into expression vector pET151. Details of plasmids preparation, sequence information, and protein expression are provided in Supplementary Note 1.

Single-molecule manipulation and data analysis. All single-molecule stretching experiments were performed using a vertical magnetic-tweezer setup ${ }^{50,52,69}$. The channel was combined with a disturbance-free, rapid solution-exchange method ${ }^{51}$ to avoid flow-drag during flow exchange. Experiments were performed in standard solution containing: 1x PBS, 1\% BSA, $2 \mathrm{mM}$ DTT, $10 \mathrm{mM}$ sodium L-ascorbate at multiple temperatures $\left(23,27,29,31,37\right.$, and $\left.47^{\circ} \mathrm{C}\right)$. The temperature was controlled by an objective heating system (Bioptechs). The details of sample preparation for single-molecule manipulation experiments are provided in Supplementary Note 2. The force-calibration of the magnetic-tweezer setup has a $10 \%$ uncertainty due to the heterogeneity of the diameter of paramagnetic beads ${ }^{69}$. Details of theoretical models of the force-extension curves of the folded/unfolded protein domain/complex and the force-dependent transition rates are provided in Supplementary Note 6 and Note 7, respectively.

Reporting summary. Further information on research design is available in the Nature Research Reporting Summary linked to this article.

\section{Data availability}

Data supporting the findings of this manuscript are available from the corresponding authors upon reasonable request. A reporting summary for this Article is available as a Supplementary Information file. Source data are provided with this paper.

Received: 2 September 2019; Accepted: 17 August 2020; Published online: 08 September 2020

\section{References}

1. Grashoff, C. et al. Measuring mechanical tension across vinculin reveals regulation of focal adhesion dynamics. Nature 466, 263-266 (2010).

2. Borghi, N. et al. E-cadherin is under constitutive actomyosin-generated tension that is increased at cell-cell contacts upon externally applied stretch. Proc. Natl Acad. Sci. USA 109, 12568-12573 (2012).

3. Austen, K. et al. Extracellular rigidity sensing by talin isoform-specific mechanical linkages. Nat. Cell Biol. 17, 1597-1606 (2015).

4. Hakonardottir, G. K. et al. In vivo quantitative analysis of Talin turnover in response to force. Mol. Biol. Cell 26, 4149-4162 (2015).

5. Chang, A. C. et al. Single molecule force measurements in living cells reveal a minimally tensioned integrin state. ACS Nano 10, 10745-10752 (2016).

6. Ringer, P. et al. Multiplexing molecular tension sensors reveals piconewton force gradient across talin-1. Nat. Methods 14, 1090-1096 (2017).

7. Tan, S. J. et al. Regulation and dynamics of force transmission at individual cell-matrix adhesion bonds. Sci. Adv. 6, eaax0317 (2020).

8. Perkins, T. T., Quake, S. R., Smith, D. E. \& Chu, S. Relaxation of a single DNA molecule observed by optical microscopy. Science 264, 822-826 (1994).

9. Smith, S. B., Cui, Y. \& Bustamante, C. Overstretching B-DNA: the elastic response of individual double-stranded and single-stranded DNA molecules. Science 271, 795-799 (1996)

10. Wang, M. D. et al. Stretching DNA with optical tweezers. Biophys. J. 72, 1335-1346 (1997)

11. Quake, S. R., Babcock, H. \& Chu, S. The dynamics of partially extended single molecules of DNA. Nature 388, 151-154 (1997).

12. Williams, M. C. \& Rouzina, I. Force spectroscopy of single DNA and RNA molecules. Curr. Opin. Struct. Biol. 12, 330-336 (2002).

13. Allemand, J. F., Bensimon, D. \& Croquette, V. Stretching DNA and RNA to probe their interactions with proteins. Curr. Opin. Struct. Biol. 13, 266-274 (2003).

14. Bryant, Z. et al. Structural transitions and elasticity from torque measurements on DNA. Nature 424, 338-341 (2003).

15. Cheng, E. et al. A pH-triggered, fast-responding DNA hydrogel. Angew. Chem. Int. Ed. Engl. 48, 7660-7663 (2009).

16. Farre, A., van der Horst, A., Blab, G. A., Downing, B. P. \& Forde, N. R. Stretching single DNA molecules to demonstrate high-force capabilities of holographic optical tweezers. J. Biophotonics 3, 224-233 (2010).

17. Wang, X. \& Ha, T. Defining single molecular forces required to activate integrin and notch signaling. Science 340, 991-994 (2013).

18. Seo, D. et al. A mechanogenetic toolkit for interrogating cell signaling in space and time. Cell 169, 1357 (2017).

19. Rosales, A. M., Vega, S. L., DelRio, F. W., Burdick, J. A. \& Anseth, K. S. Hydrogels with reversible mechanics to probe dynamic cell microenvironments. Angew. Chem. Int. Ed. Engl. 56, 12132-12136 (2017). 
20. Brazin, K. N. et al. The T cell antigen receptor alpha transmembrane domain coordinates triggering through regulation of bilayer immersion and CD3 subunit associations. Immunity 49, 829-841 e826 (2018).

21. Xing, Z. et al. Microrheology of DNA hydrogels. Proc. Natl Acad. Sci. USA 115, 8137-8142 (2018).

22. Bera, S. et al. Rigid helical-like assemblies from a self-aggregating tripeptide. Nat. Mater. 18, 503-509 (2019).

23. Shaevitz, J. W., Abbondanzieri, E. A., Landick, R. \& Block, S. M. Backtracking by single RNA polymerase molecules observed at near-base-pair resolution. Nature 426, 684-687 (2003).

24. Spadaro, D. et al. Tension-dependent stretching activates ZO-1 to control the junctional localization of its interactors. Curr. Biol. 27, 3783-3795 e3788 (2017).

25. Yu, M. et al. mDial senses both force and torque during F-actin filament polymerization. Nat. Commun. 8, 1650 (2017).

26. Le, S. et al. Bacillus subtilis RecA with DprA-SsbA antagonizes RecX function during natural transformation. Nucleic Acids Res. 45, 8873-8885 (2017).

27. Yuan, G. et al. Elasticity of the transition state leading to an unexpected mechanical stabilization of titin immunoglobulin domains. Angew. Chem. Int. Ed. Engl. 56, 5490-5493 (2017)

28. Le, S. et al. Dystrophin as a molecular shock absorber. ACS Nano 12, 12140-12148 (2018).

29. Chivers, C. E. et al. A streptavidin variant with slower biotin dissociation and increased mechanostability. Nat. Methods 7, 391-393 (2010).

30. Chivers, C. E., Koner, A. L., Lowe, E. D. \& Howarth, M. How the biotinstreptavidin interaction was made even stronger: investigation via crystallography and a chimaeric tetramer. Biochem. J. 435, 55-63 (2011).

31. Chen, H. et al. Dynamics of equilibrium folding and unfolding transitions of titin immunoglobulin domain under constant forces. J. Am. Chem. Soc. 137, 3540-3546 (2015)

32. Jegou, A., Carlier, M. F. \& Romet-Lemonne, G. Formin mDial senses and generates mechanical forces on actin filaments. Nat. Commun. 4, 1883 (2013).

33. Yu, M. et al. Effects of mechanical stimuli on profilin- and formin-mediated actin polymerization. Nano Lett. 18, 5239-5247 (2018)

34. Mason, J. M. \& Arndt, K. M. Coiled coil domains: stability, specificity, and biological implications. Chembiochem 5, 170-176 (2004).

35. Lapenta, F., Aupic, J., Strmsek, Z. \& Jerala, R. Coiled coil protein origami: from modular design principles towards biotechnological applications. Chem. Soc. Rev. 47, 3530-3542 (2018).

36. Florin, E., Moy, V. \& Gaub, H. Adhesion forces between individual ligandreceptor pairs. Science 264, 415-417 (1994).

37. Neuert, G., Albrecht, C., Pamir, E. \& Gaub, H. E. Dynamic force spectroscopy of the digoxigenin-antibody complex. FEBS Lett. 580, 505-509 (2006).

38. Jadhav, V. S., Bruggemann, D., Wruck, F. \& Hegner, M. Single-molecule mechanics of protein-labelled DNA handles. Beilstein J. Nanotechnol. 7 , 138-148 (2016).

39. Clausen-Schaumann, H., Rief, M., Tolksdorf, C. \& Gaub, H. E. Mechanical stability of single DNA molecules. Biophys. J. 78, 1997-2007 (2000).

40. Guo, S., Efremov, A. K. \& Yan, J. Understanding the catch-bond kinetics of biomolecules on a one-dimensional energy landscape. Commun. Chem. 2, 30 (2019).

41. Harbury, P. B., Zhang, T., Kim, P. S. \& Alber, T. A switch between two-, three-, and four-stranded coiled coils in GCN4 leucine zipper mutants. Science 262, 1401-1407 (1993)

42. Burkhard, P., Stetefeld, J. \& Strelkov, S. V. Coiled coils: a highly versatile protein folding motif. Trends Cell Biol. 11, 82-88 (2001).

43. Thomas, F., Boyle, A. L., Burton, A. J. \& Woolfson, D. N. A set of de novo designed parallel heterodimeric coiled coils with quantified dissociation constants in the micromolar to sub-nanomolar regime. J. Am. Chem. Soc. 135 5161-5166 (2013).

44. Bornschlogl, T. \& Rief, M. Single-molecule dynamics of mechanical coiled-coil unzipping. Langmuir 24, 1338-1342 (2008).

45. Goktas, M. et al. Molecular mechanics of coiled coils loaded in the shear geometry. Chem. Sci. 9, 4610-4621 (2018).

46. Reverberi, R. \& Reverberi, L. Factors affecting the antigen-antibody reaction. Blood Transfus. 5, 227-240 (2007)

47. Lo, Y.-S., Simons, J. \& Beebe, T. P. Temperature dependence of the biotin -avidin bond-rupture force studied by atomic force microscopy. J. Phys. Chem. B 106, 9847-9852 (2002).

48. Thompson, K. E., Bashor, C. J., Lim, W. A. \& Keating, A. E. SYNZIP protein interaction toolbox: in vitro and in vivo specifications of heterospecific coiledcoil interaction domains. ACS Synth. Biol. 1, 118-129 (2012).

49. Chen, Z. et al. Programmable design of orthogonal protein heterodimers. Nature 565, 106-111 (2019).

50. Chen, $H$. et al. Improved high-force magnetic tweezers for stretching and refolding of proteins and short DNA. Biophys. J. 100, 517-523 (2011).

51. Le, S. et al. Disturbance-free rapid solution exchange for magnetic tweezers single-molecule studies. Nucleic Acids Res. 43, e113 (2015).
52. Le, S., Liu, R., Lim, C. T. \& Yan, J. Uncovering mechanosensing mechanisms at the single protein level using magnetic tweezers. Methods 94, 13-18 (2016)

53. Yu, M. et al. Force-dependent regulation of talin-KANK1 complex at focal adhesions. Nano Lett. 19, 5982-5990 (2019).

54. Guo, S. et al. Structural-elastic determination of the force-dependent transition rate of biomolecules. Chem. Sci. 9, 5871-5882 (2018)

55. Le, S. et al. Mechanotransmission and mechanosensing of human alphaactinin 1. Cell Rep. 21, 2714-2723 (2017).

56. Nedelec, F. J., Surrey, T., Maggs, A. C. \& Leibler, S. Self-organization of microtubules and motors. Nature 389, 305-308 (1997).

57. Sakamoto, T., Webb, M. R., Forgacs, E., White, H. D. \& Sellers, J. R. Direct observation of the mechanochemical coupling in myosin Va during processive movement. Nature 455, 128-132 (2008)

58. Chen, L., Nakamura, M., Schindler, T. D., Parker, D. \& Bryant, Z. Engineering controllable bidirectional molecular motors based on myosin. Nat. Nanotechnol. 7, 252-256 (2012)

59. Melli, L. et al. Bipolar filaments of human nonmuscle myosin 2-A and 2-B have distinct motile and mechanical properties. Elife 7, https://doi.org/ 10.7554/eLife.32871 (2018).

60. Yao, M. et al. The mechanical response of talin. Nat. Commun. 7, 11966 (2016).

61. Giannone, G. et al. Periodic lamellipodial contractions correlate with rearward actin waves. Cell 116, 431-443 (2004).

62. Galior, K., Liu, Y., Yehl, K., Vivek, S. \& Salaita, K. Titin-based nanoparticle tension sensors map high-magnitude integrin forces within focal adhesions. Nano Lett. 16, 341-348 (2016).

63. Jurchenko, C., Chang, Y., Narui, Y., Zhang, Y. \& Salaita, K. S. Integringenerated forces lead to streptavidin-biotin unbinding in cellular adhesions. Biophys. J. 106, 1436-1446 (2014)

64. Wang, Y., Wang, H., Tran, M. V., Algar, W. R. \& Li, H. Yellow fluorescent protein-based label-free tension sensors for monitoring integrin tension. Chem. Commun. 56, 5556-5559 (2020).

65. Coleman, T. R. \& Mooseker, M. S. Effects of actin filament cross-linking and filament length on actin-myosin interaction. J. Cell Biol. 101, 1850-1857 (1985).

66. Mogilner, A. \& Oster, G. Cell motility driven by actin polymerization. Biophys. J. 71, 3030-3045 (1996)

67. Svitkina, T. The Actin Cytoskeleton and Actin-Based Motility. Cold Spring Harb. Perspect. Biol. 10, https://doi.org/10.1101/cshperspect.a018267 (2018).

68. Zakeri, B. et al. Peptide tag forming a rapid covalent bond to a protein, through engineering a bacterial adhesin. Proc. Natl Acad. Sci. USA 109 E690-E697 (2012).

69. Zhao, X., Zeng, X., Lu, C. \& Yan, J. Studying the mechanical responses of proteins using magnetic tweezers. Nanotechnology 28, 414002 (2017).

\section{Acknowledgements}

We thank Ms. Hongying Chen (protein cloning expression core, MBI) for her help on the protein expression and Mr. Andrew Wong (scientific communication core, MBI) for proofreading the manuscript. The research is funded by the Singapore Ministry of Education Academic Research Fund Tier 2 (MOE2019-T2-1-099 to J.Y.), National Research Foundation, Prime Minister's Office, Singapore, under its NRF Investigatorship Program (NRF Investigatorship Award NRF-NRFI2016-03 to J.Y.), and the Ministry of Education under the Research Centers of Excellence program through the Mechanobiology Institute at National University of Singapore (to J.Y.).

\section{Author contributions}

J.Y., M.Y., and S.L. designed the study. M.Y., and S.L., performed the single-molecule stretching experiments and analyzed the data; Z.Z. and Z.C. provided technological support; J. Y., M.Y., and S.L. interpreted the data and wrote the paper.

\section{Competing interests}

The authors declare no competing interests.

\section{Additional information}

Supplementary information is available for this paper at https://doi.org/10.1038/s41467 020-18323-w.

Correspondence and requests for materials should be addressed to S.L. or J.Y.

Peer review information Nature Communications thanks the anonymous reviewers for their contributions to the peer review of this work.

Reprints and permission information is available at http://www.nature.com/reprints

Publisher's note Springer Nature remains neutral with regard to jurisdictional claims in published maps and institutional affiliations. 
(c) (i) Open Access This article is licensed under a Creative Commons Attribution 4.0 International License, which permits use, sharing, adaptation, distribution and reproduction in any medium or format, as long as you give appropriate credit to the original author(s) and the source, provide a link to the Creative Commons license, and indicate if changes were made. The images or other third party material in this article are included in the article's Creative Commons license, unless indicated otherwise in a credit line to the material. If material is not included in the article's Creative Commons license and your intended use is not permitted by statutory regulation or exceeds the permitted use, you will need to obtain permission directly from the copyright holder. To view a copy of this license, visit http://creativecommons.org/ licenses/by/4.0/.

(C) The Author(s) 2020 